\title{
Caracterização reológica de suspensões argilosas salinas com goma xantana para fluidos de perfuração de poços de petróleo
}

\author{
Rheological characterization of saline \\ clay dispersions with xanthan gum for oil \\ well drilling fluids
}

Gabriel Sahade de Souza ${ }^{1}$, Samuel Luporini ${ }^{2}$, Isabel Cristina Rigoli ${ }^{3}$

\footnotetext{
${ }^{1}$ Petróleo Brasileiso S.A. - Petrobras. Universidade Petrobras. Av. Antônio Carlos Magalhães. 1113 - 41850-903 - Pituba, Salvador, BA.

e-mail: gabrielsahade@petrobras.com.br

${ }^{2}$ Programa de Pós graduação em Engenharia Química, Departamento de Engenharia Química, Universidade Federal da Bahia, R. Prof. Aristídes Novis, 2 - 40210-630 - Federação, Salvador, BA.

e-mail: samluporini@terra.com.br

${ }^{3}$ Instituto de Química, Departamento de Química Inorgânica, Universidade Federal da Bahia, Rua Barão de Jeremoabo, 147 Campus Universitário de Ondina. 40.170-115, Salvador, BA.

e-mail: irigoli@ufba.br
}

\section{RESUMO}

Testes de cisalhamento utilizando um viscosímetro de cilindros coaxiais foram realizados no intuito de se determinar as propriedades reológicas de dispersões de goma xantana com bentonita sódica em presença de diferentes concentrações de cloreto de sódio $(\mathrm{NaCl})$ e um valor fixo de concentração de cloreto de cálcio $\left(\mathrm{CaCl}_{2}\right)$ à temperatura ambiente. A reologia das dispersões com diferentes concentrações de $\mathrm{NaCl}$ e bentonita sódica foram preparadas fixando as concentrações de goma xantana e $\mathrm{CaCl}_{2}$ em 0,47\% e 0,455\% respectivamente (ambas as porcentagens em massa) e utilizando água deionizada como meio dispersivo. As dispersões mostraram um bom ajuste ao modelo reológico de potência modificado (Herschel-Bulkley) através de regressões não lineares. Foi feito um Planejamento Composto Central (PCC) em dois níveis no qual as variáveis independentes foram as concentrações de bentonita sódica e cloreto de sódio e as variáveis dependentes foram o limite de escoamento $\left(\tau_{0}\right)$, o índice de consistência $(k)$ e o índice de fluxo $(n)$. As amostras foram preparadas sob agitação com 24 horas de antecedência e as medições dos pontos da curva reológica foram obtidas através do viscosímetro Haake. Os resultados da análise mostraram que a tensão limite de escoamento é fortemente influenciada pela concentração de bentonita enquanto que os índices de consistência e fluxo são influenciados tanto pela concentração de bentonita como pela concentração do cloreto de sódio. Esses resultados mostram que é possível melhorar o desempenho de fluidos de perfuração argilosos através da manipulação do teor de sais e argila no mesmo.

Palavras-chave: Fluidos de perfuração, bentonita, reologia.

\section{ABSTRACT}

Shear tests using a coaxial cylinders viscometer were performed in order to determine the rheological properties of xanthan gum dispersions with sodium bentonite in the presence of different concentrations of sodium chloride $(\mathrm{NaCl})$ and a fixed concentration of calcium chloride $\left(\mathrm{CaCl}_{2}\right)$ at room temperature. The dispersions rheology with different concentrations of $\mathrm{NaCl}$ and sodium bentonite were prepared by fixing the xanthan gum and $\mathrm{CaCl}_{2}$ concentrations of $0.47 \%$ and $0.455 \%$ respectively (both mass percentages) using deionized water. The dispersions showed a good fit to modified power rheological model (Herschel-Bulkley) by nonlinear regressions. A Central Composite Design was made for two levels. The independent variables were sodium bentonite and sodium chloride concentrations and the dependent variables were the yield point $\left(\tau_{0}\right)$, the consistency index $(\mathrm{k})$ and the flow index $(\mathrm{n})$. Samples were prepared with stirring 24 hours in advance and the measurement of the rheological curve points were obtained by Haake viscometer. The results of the 
analysis showed that the yield stress is strongly influenced by the concentration of bentonite while the consistency and flow indexes are influenced by both sodium chloride and sodium bentonite concentrations. These results show that it is possible to improve the performance of clay drilling fluids through manipulation of salts and clay content.

Keywords: Drilling fluids, bentonite, rheology.

\section{INTRODUÇÃO}

A perfuração de poços é um processo que tem como objetivo não apenas à produção de óleo e gás natural, como é o caso dos poços produtores, mas também a injeção de fluidos que auxiliem na recuperação de hidrocarbonetos. Os poços podem ser perfurados tanto em terra (onshore) como em mar (offshore).

No cenário nacional atual, a maior parte das reservas descobertas de óleo e gás encontra-se em reservatórios cujo acesso só é possível através da perfuração de poços submarinos. Esse tipo de cenário requer que novas tecnologias sejam alcançadas no intuito de atender as novas necessidades. Essas necessidades são decorrentes da perfuração de poços de geometria complexa em diferentes tipos de formações. O fluido de perfuração possui um papel essencial no atingimento dessa meta, de modo que ele deve atender as demandas operacionais, ambientais e de custos.

Os fluidos de perfuração são misturas complexas que, do ponto de vista químico, podem assumir aspectos de suspensão, dispersão coloidal ou emulsão, dependendo do estado físico dos componentes [1]. Eles são tradicionalmente classificados de acordo com a sua base, que pode ser aquosa (FBA), não aquosa (FBNA), ou gasosa. Os fluidos de perfuração de base não aquosa também são conhecidos como fluidos de base orgânica sintética (SBM, Synthetic Base Mud).

Atualmente os fluidos de perfuração têm sido alvo de diversas discussões entre pesquisadores, empresas do ramo de petróleo e órgãos ambientais. O principal ponto de discussão é encontrar soluções aos problemas causados pela contaminação do meio ambiente e das formações geológicas portadoras de hidrocarbonetos ou de água (aquíferos) com as quais o fluido de perfuração entra em contato. O FBA é, de um modo geral, muito menos agressivo ao meio ambiente do que o FBNA, sendo geralmente descartado no mar, quando em perfuração de poços marítimos, dentro dos requisitos pré-estabelecidos pela legislação vigente.

As principais funções dos fluidos de perfuração são: remover e transportar os fragmentos de rocha perfurados (cascalhos), controlar as pressões da formação, manter a estabilidade do poço e resfriar a broca durante a perfuração. As propriedades reológicas dos fluidos de perfuração são de grande relevância para que o mesmo desempenhe de maneira eficiente as suas funções [2].

A bentonita é um tipo de argila rica em esmectita, argilomineral responsável pelo aumento da viscosidade das dispersões. Ela é utilizada em fluidos de perfuração de base aquosa com o objetivo de controlar as propriedades reológicas e de filtração. Um aumento da viscosidade do fluido de perfuração está intimamente ligado a um bom transporte de cascalhos do fundo do poço para a superfície. No entanto, o uso da bentonita como viscosificante deve ser feito com cautela, pois altas concentrações desse aditivo podem causar problemas durante a perfuração, como por exemplo, aumento das chances de prisão de coluna por diferencial de pressão e aumento de torque da coluna por conta do atrito [3].

A grande maioria das partículas com dimensões coloidais apresenta uma carga superficial residual. No caso dos argilominerais, essa carga é resultado das substituições isomórficas. Quando dispersas num líquido polar, a carga superficial dessas partículas pode ser eletricamente neutralizada através de contra-íons existentes na solução, formando a conhecida dupla camada elétrica [3].

De modo simplificado, a dupla camada elétrica é composta por uma camada fixa de íons (mais fortemente ligados à superfície) e uma camada difusa de íons (menos fortemente ligada). Uma vez em equilíbrio, todas as partículas do sistema adquirem a mesma carga superficial, assim sendo, é o processo de repulsão eletrostática o fator responsável pela manutenção da estabilidade do sistema. Observa-se que quando é reduzida a repulsão entre as partículas, há a propensão de ocorrência dos processos de floculação, enquanto que quando a carga superficial das partículas é aumentada, o sistema permanece em estado disperso.

Para aumentar a viscosidade do fluido sem aumentar a concentração de bentonita, utiliza-se em conjunto outros aditivos poliméricos viscosificantes. Um dos principais polímeros utilizados em conjunto com a bentonita é a goma xantana. A goma xantana é um biopolímero utilizado na indústria do petróleo que tem como função aumentar a viscosidade de fluidos de perfuração de base aquosa mesmo em condições de altas temperaturas, salinidade e pressão. Sua alta eficiência frente a condições de altas temperaturas e salinidade torna a goma xantana um dos viscosificantes mais utilizados em fluidos de perfuração de base aquosa [4]. Por ser um viscosificante mais caro do que a bentonita, a goma xantana é utilizada em concentrações tão peque- 
nas quanto $0,5 \%$ (em massa) em conjunto com a argila.

De um modo geral, as soluções de goma xantana são estáveis na presença da maioria dos sais. Sob determinadas condições de temperatura e pressão, íons divalentes podem causar precipitação do polímero. Essa precipitação pode ocorrer devido à interação do cátion com parte carregada negativamente da carbonila. Como a concentração de sal afeta a viscosidade de uma solução de goma xantana depende da concentração do polímero. Em concentrações mássicas de goma menores do que $0,5 \%$ (em massa), sais monovalentes, como o cloreto de sódio, causam uma pequena redução da viscosidade [4].

Um dos modelos reológicos mais simples que melhor caracteriza as dispersões argilosas estudadas é o modelo reológico de Herschel-Bulkley, também conhecido como modelo de potência modificado. Trata-se de um modelo que caracteriza o fluxo de fluidos não newtonianos a partir de três parâmetros: a tensão limite de escoamento $\left(\tau_{0}\right)$, o índice de consistência $(\mathrm{k})$ e o índice de fluxo (n) conforme mostrado na Equação 1 :

$$
\tau=\tau_{0}+k \gamma^{n}
$$

Onde $\tau$ é a tensão cisalhante e $\gamma$ é a taxa de cisalhamento. Por trabalhar com três parâmetros, os dados experimentais geralmente se ajustam melhor a esse modelo, sendo um dos modelos reológicos mais utilizados na indústria do petróleo para caracterizar o escoamento dos fluidos de perfuração [5].

A tensão limite de escoamento, ou simplesmente limite de escoamento $\left(\tau_{0}\right)$ está diretamente relacionado com as forças de interação entre as partículas dispersas no meio. Trata-se de um parâmetro reológico que representa a tensão mínima necessária para que o fluido inicie o escoamento. Caso seja aplicada uma tensão menor do que a tensão limite, o fluido se comportará como um sólido. Esse parâmetro é importante para fluidos de perfuração, uma vez que evita a decantação dos cascalhos no fundo do poço quando o fluido encontrase na estática. Quanto maior o valor de $\tau_{0}$, menor o risco de decantação de cascalhos no poço [3].

O índice de consistência indica o grau de resistência ao escoamento do fluido, ou seja, quanto maior o valor de $k$, mais viscoso será o fluido. Um alto valor de $k$ facilita o carreamento dos cascalhos do fundo do poço até a superfície durante a perfuração ou circulação do fluido dentro do poço [3,5].

O índice de comportamento ( $n$ ) indica o grau de afastamento do fluido do modelo newtoniano. Quando o valor de $n$ é menor do que a unidade, temos um fluido pseudoplástico e quando ele é maior do que a unidade temos um fluido dilatante. Fluidos pseudoplásticos apresentam redução da viscosidade com o aumento da taxa de cisalhamento (vazão), o que é interessante para o fluido de perfuração, pois menores valores de viscosidades implicam em menores valores de perda de carga no poço $[3,5]$.

O presente trabalho faz um estudo do comportamento reológico de suspensões argilosas salinas com goma xantana a partir de um planejamento experimental composto central (PCC). O objetivo desse estudo é verificar a influência da concentração de bentonita sódica e da concentração de cloreto de sódio nos parâmetros reológicos de suspensões de argila com goma xantana no intuito de melhorar o desempenho de fluidos de perfuração de base aquosa argilosos.

\section{MATERIAIS E MÉTODOS}

\subsection{Materiais}

Os reagentes utilizados para a preparação das amostras de fluidos foram: bentonita sódica (Drescon S.A. DRESCONPEL), goma xantana (Sigma Aldrich, USA), cloreto de sódio P.A. (C.A.Q. - Casa das Químicas Ind.), cloreto de cálcio dihidratado P.A. (C.A.Q. - Casa das Químicas Ind.) e água deionizada. Os equipamentos e vidrarias utilizados foram: um béquer de $150 \mathrm{~mL}$, uma balança analítica, um agitador mecânico (PROLAB), um refrigerador e um viscosímetro Haake Rehotest (Medingen 2.1).

As especificações da bentonita utilizada no que se refere ao teor de umidade, granulometria e pH são mostradas na Tabela 1.

Tabela 1: Especificações da bentonita sódica utilizada.

\begin{tabular}{l|l}
\hline Características & Valores médios encontrados \\
\hline Umidade $(\%)$ & $9,0-14,0$ \\
\hline Retido na Peneira ABNT $75 \mu \mathrm{m}(\%$ em peso $)$ & $4,0-10,0$ \\
\hline $\mathrm{pH}$ & $9,0-10,5$ \\
\hline
\end{tabular}




\subsection{Preparação das amostras de fluido e medição das propriedades}

As soluções foram preparadas de tal forma que as concentrações de goma xantana e de cloreto de cálcio foram mantidas constantes em $0,47 \%$ e $0,455 \%$ (em massa) respectivamente. A temperatura também foi mantida constante em $25^{\circ} \mathrm{C}$.

$\mathrm{O}$ valor de $0,47 \%$ representa um valor médio de concentração (em massa) de goma xantana normalmente encontrado em fluidos de perfuração argilosos nos quais o polímero é utilizado em conjunto com a bentonita como viscosificante.

Já o valor de $0,455 \%$ do cloreto de cálcio representa a concentração (em massa) média desse sal encontrada no fluido de perfuração. As principais fontes do íon $\mathrm{Ca}^{2+}$ presentes nos fluidos de perfuração de base aquosa são a perfuração de formações carbonáticas e a perfuração de cimento proveniente de operações de cimentação de revestimentos dentro do poço.

A preparação das amostras seguiu o procedimento sugerido por SHIROMA [6]. Os componentes foram adicionados em um béquer de $150 \mathrm{~mL}$ sob agitação a uma velocidade constante de $1.500 \mathrm{rpm}$ num agitador mecânico do tipo Prolab. Inicialmente foi adicionada, em pequenas quantidades, a goma xantana em água deionizada durante 5 minutos. Após a adição de goma xantana, aguardou-se sob agitação por 15 minutos para maximizar a hidratação do polímero.

Após a hidratação da goma, ainda sob agitação, por mais 5 minutos, foi adicionada, em pequenas quantidades, a massa correspondente de bentonita sódica ainda sob agitação e posteriormente, do mesmo modo, aguardou-se 15 minutos. Depois da adição da bentonita, adicionou-se o cloreto de sódio também em pequenas quantidades durante 5 minutos até que houvesse a sua total dissolução e aguardou-se por mais 10 minutos para a homogeneização do sistema.

Por fim, adicionou-se a quantidade correspondente de cloreto de cálcio por 3 minutos ainda sob agitação e agitou-se por mais 15 minutos, de modo que, no final, a dispersão tinha um volume de $100 \mathrm{~mL}$. Após o preparo das dispersões, deixou-se a mesma em repouso por 24 horas em uma temperatura de $4{ }^{\circ} \mathrm{C}$. SHIROMA [6], que trabalhou com a bentonita sódica com a mesma faixa granulométrica, mediu as propriedades reológica das dispersões argilosas com o tempo e mostrou que o período de $24 \mathrm{~h}$ de hidratação é suficiente para se obter os valores otimizados dos parâmetros reológicos.

A realização das medições no viscosímetro Haake seguiu as orientações do manual do equipamento. O aparelho opera com doze modos de velocidade e as taxas de cisalhamento trabalhadas pelo viscosímetro variaram de aproximadamente 4 a $1890 \mathrm{~s}^{-1}$ conforme pode ser visto no Anexo do trabalho. O rotor utilizado foi o spindle S625 (raio $=3,90.10^{-2} \mathrm{~m}$ ) e o cilindro externo com raio de $4,00.10^{-2} \mathrm{~m}$. Cada rotação do viscosímetro é correspondente a uma taxa de cisalhamento conforme o manual do equipamento.

O procedimento de medição consistiu em uma série de etapas. Inicialmente houve a transferência da amostra do béquer para o interior do cilindro externo, no qual foram realizadas as medições. Ajustou-se o nível do viscosímetro para que o rotor ficasse completamente submerso no fluido de acordo com a marca do cilindro externo. Para iniciar as medições das tensões de cisalhamento, selecionou-se a velocidade de rotação para o menor valor (menor taxa de cisalhamento). Aguardou-se que o indicador de tensão ficasse estável, indicando que atingiu-se o estado estacionário e anotou-se o valor de tensão obtido. Sucessivamente fez-se as leituras das tensões nas demais rotações (demais taxas de cisalhamento), alterando velocidade do motor através da alavanca do viscosímetro. A cada troca de amostra de fluido, o equipamento foi limpo.

Ao final, foram obtidos valores de tensão versus taxa de cisalhamento nas diversas rotações do viscosímetro. Com os pontos de taxa de cisalhamento versus tensão fez-se uma regressão não linear dos dados pelo método Quasi-Newton BFGS (proposto por Broyden, Fletcher, Goldfarb e Shanno), utilizando o software STATISTICA versão 7.0 para cada amostra para a obtenção dos parâmetros reológicos da Equação 1. Os pontos das curvas com os seus parâmetros e com os respectivos coeficientes de determinação $\left(\mathrm{R}^{2}\right)$ foram obtidos a partir do trabalho de SOUZA [7] e podem ser vistas no Anexo deste trabalho.

\subsection{Metodologia do Planejamento Composto Central}

Para analisar a influência da bentonita sódica e do cloreto de sódio na dispersão, foi realizado um Planejamento Composto Central (PCC) [8] com dois fatores no qual as variáveis dependentes, ou variáveis resposta, foram os índices de consistência $(k)$ e de comportamento $(n)$ e a tensão limite de escoamento $\left(\tau_{0}\right)$, parâmetros do modelo de Herschel-Bulkley.

Foi empregado um modelo matemático de superfície de resposta para o estudo das variáveis independentes: concentrações de bentonita sódica e de cloreto de sódio. O experimento foi realizado com oito pontos centrais. As repetições no ponto central têm duas finalidades: fornecer uma medida do erro puro e estabilizar 
a variância da resposta prevista. O software utilizado para a análise estatística dos dados foi o STATISTICA versão 7.0.

As variáveis independentes foram estudadas em cinco níveis codificados $(-\sqrt{2},-1,0,+1,+\sqrt{2})$. A codificação das variáveis de entrada pode ser vista na Tabela 2 . As faixas de concentrações de bentonita sódica e de cloreto de sódio bem como os valores fixados de concentrações de cloreto de cálcio $(0,455 \%)$ e goma xantana $(0,47 \%)$ foram escolhidos a partir de valores usuais encontrados nos fluidos durante as operações de perfuração.

Tabela 2: Níveis das variáveis independentes para o Planejamento Composto Central (PCC).

\begin{tabular}{l|l|l}
\hline Nível & Concentração de Bentonita (\%) & Concentração de NaCl (\%) \\
\hline$-\sqrt{2}$ & 1,34 & 5,76 \\
\hline-1 & 3 & 7 \\
\hline 0 & 7 & 10 \\
\hline+1 & 11 & 13 \\
\hline$+\sqrt{2}$ & 12,66 & 14,34 \\
\hline
\end{tabular}

Uma das maneiras mais confiáveis de se avaliar a qualidade do ajuste do modelo gerado é empregando a análise de variância (ANOVA). Na ANOVA a variação total da resposta é definida como a soma quadrática de dois componentes: a soma quadrática da regressão $\left(\mathrm{SQ}_{\mathrm{reg}}\right)$ e a soma quadrática dos resíduos $\left(\mathrm{SQ}_{\mathrm{res}}\right)$. A soma quadrática da variação total, corrigida para a média ( $\left.\mathrm{SQ}_{\text {total }}\right)$ pode ser escrita como a Equação 2 [8].

$$
S Q_{\text {total }}=S Q_{\text {reg }}+S Q_{\text {res }}
$$

Quando algum modelo é ajustado aos dados, a soma quadrática do erro puro é sempre uma parte da soma quadrática dos resíduos $\left(\mathrm{SQ}_{\mathrm{res}}\right)$. Logo, a $\mathrm{SQ}_{\mathrm{res}}$ pode ser definida segundo a Equação 3.

$$
\sum_{i=1}^{m} \sum_{j=1}^{r_{i}}\left(y_{i j}-\widehat{y}_{l}\right)^{2}=\sum_{i=1}^{m} \sum_{j=1}^{r_{i}}\left(y_{i j}-\bar{y}_{l}\right)^{2}+\sum_{i=1}^{m}\left(\widehat{y}_{l}-\bar{y}_{l}\right)^{2}
$$

Da Equação 3, $m$ é o número total de níveis do planejamento, isto é, pontos experimentais do planejamento; $\widehat{y}_{l}$ é o valor estimado pelo modelo para o nível $i$ e $\bar{y}_{l}$ é o valor médio das replicatas $(r)$. De maneira simplificada, podemos escrever a Equação 3 da seguinte forma:

$$
S Q_{\text {res }}=S Q_{e p}+S Q_{f a j}
$$

$\mathrm{O}$ primeiro termo da parte direita da Equação 4 é chamado de soma quadrática do erro puro $\left(\mathrm{SQ}_{\mathrm{ep}}\right) \mathrm{e}$ está relacionado exclusivamente com os erros aleatórios das replicatas, ou seja, ele está diretamente relacionado ao desvio dos dados em torno da média. Já o segundo termo da parte direita da equação é chamado de soma quadrática da falta de ajuste $\left(\mathrm{SQ}_{\mathrm{faj}}\right)$, pois ele é uma medida da discrepância entre a resposta do modelo de previsão $\left(\widehat{y}_{l}\right)$ e a média das replicatas $\left(\bar{y}_{l}\right)$ realizadas no conjunto de condições experimentais.

Em termos práticos, um bom modelo necessita ter uma regressão significativa e uma falta de ajuste não significativa. Da variação que fica com os resíduos, espera-se que a maior parte fique com o erro puro, que é o erro experimental, e não com a falta de ajuste, que está diretamente relacionada ao modelo.

Um dos parâmetros utilizados para observar se todos os desvios em torno da média foram explicados pela regressão é o coeficiente de determinação $\mathrm{R}^{2}$ (Equação 5). $\mathrm{O}$ valor de $\mathrm{R}^{2}$ representa a fração da variação que é explicada pela falta de ajuste do modelo. Quanto mais próximo de 1 o valor do coefíciente estiver, melhor será o ajuste do modelo às respostas observadas.

$$
R^{2}=\frac{S Q_{\text {res }}}{S Q_{\text {total }}}
$$

É importante salientar que o erro puro não explica o modelo, então o máximo valor possível de $\mathrm{R}^{2}\left(\mathrm{R}^{2}\right.$ máx.) é:

$$
R_{(\text {máx.) }}^{2}=\frac{S Q_{t o t a l}-S Q_{e p}}{S Q_{\text {total }}}
$$




\section{RESULTADOS E DISCUSSÕES}

O planejamento experimental completo pode ser observado na Tabela 3. Os valores de tensão limite de escoamento $\left(\tau_{0}\right)$ e de índice de consistência $(k)$ estão em $\mathrm{Pa}$ e Pa.s ${ }^{n}$ respectivamente, enquanto que o índice de comportamento $(n)$ é adimensional.

Foi realizada uma análise separada de cada um dos parâmetros a fim de facilitar a compreensão dos resultados gerados.

Tabela 3: Níveis das variáveis independentes para o Planejamento Composto Central (PCC).

\begin{tabular}{l|l|l|l|l|l}
\hline Fluido & Bentonita sódica & Cloreto de Sódio & $\boldsymbol{\tau}_{\boldsymbol{0}}$ & $\boldsymbol{k}$ & $\boldsymbol{n}$ \\
\hline 1 & -1 & -1 & 3,167 & 0,874 & 0,458 \\
\hline 2 & -1 & +1 & 1,298 & 0,816 & 0,455 \\
\hline 3 & +1 & -1 & 4,207 & 2,489 & 0,392 \\
\hline 5 & +1 & +1 & 5,996 & 1,903 & 0,424 \\
\hline 6 & $-\sqrt{2}$ & 0 & 2,030 & 0,609 & 0,485 \\
\hline 7 & $+\sqrt{2}$ & 0 & 6,864 & 2,494 & 0,410 \\
\hline 9 & 0 & $-\sqrt{2}$ & 4,520 & 1,703 & 0,401 \\
\hline 10 & 0 & $+\sqrt{2}$ & 3,233 & 0,863 & 0,483 \\
\hline 11 & 0 & 0 & 3,005 & 1,580 & 0,351 \\
\hline 13 & 0 & 0 & 2,700 & 1,513 & 0,358 \\
\hline 14 & 0 & 0 & 3,796 & 1,859 & 0,341 \\
\hline 15 & 0 & 0 & 3,282 & 1,442 & 0,364 \\
\hline 16 & 0 & 0 & 2,794 & 1,682 & 0,347 \\
\hline
\end{tabular}

\subsection{Análise da tensão limite de escoamento $\left(\tau_{0}\right)$}

A Tabela 4 apresenta os valores da análise de variância (ANOVA) para a variável de resposta limite de escoamento $\left(\tau_{0}\right)$, onde também é apresentado o valor do coeficiente de determinação $\left(\mathrm{R}^{2}\right)$.

Tabela 4: Análise de variância (ANOVA) para a variável limite de escoamento $\left(\tau_{0}\right)$ relativa ao PCC.

\begin{tabular}{l|l|l|l|l|l}
\hline Fator analisado & Soma dos Quadrados & Graus de Liberdade & Quadrado Médio & F & p \\
\hline Bentonita (Linear) & 19,767 & 1 & 19,767 & 103,272 & 0,000 \\
\hline Bentonita (Quad.) & 2,407 & 1 & 2,407 & 12,575 & 0,005 \\
\hline $\mathrm{NaCl}$ (Linear) & 0,451 & 1 & 0,451 & 2,3576 & 0,156 \\
\hline $\mathrm{NaCl}$ (Quad.) & 0,554 & 1 & 0,555 & 2,897 & 0,120 \\
\hline Bentonita x NaCl & 3,344 & 1 & 3,344 & 17,468 & 0,002 \\
\hline Erro Puro & 0,896 & 7 & 0,128 & $\mathbf{R}^{\mathbf{2}}$ (máx.) & $\mathbf{R}^{\mathbf{2}}$ \\
\hline Falta de Ajuste & 1,018 & 3 & 0,339 & & \multirow{2}{*}{0,932} \\
\hline Total & 28,438 & 15 & - & 0,968 & \multirow{2}{*}{} \\
\hline
\end{tabular}

A partir de uma regressão multivariada, podemos chegar numa equação para calcular o valor de $\tau_{0}$ a partir das variáveis independentes codificadas na Equação 7.

$\tau_{0}=B_{0}+B_{1}$ Bent $+B_{11}$ Bent $^{2}+B_{2} \mathrm{NaCl}+B_{22} \mathrm{NaCl}^{2}+B_{12}$ Bent. NaCl

$\mathrm{O}$ valor de $\tau_{0}$ é dado em Pa. Os valores dos coeficientes são mostrados na Tabela 5. 
Tabela 5: Coeficientes de regressão para a variável limite de escoamento $\left(\tau_{0}\right)$.

\begin{tabular}{l|l}
\hline Coeficiente & Valor \\
\hline$B_{0}$ & 3,103 \\
\hline$B_{1}$ & 1,572 \\
\hline$B_{11}$ & 0,549 \\
\hline$B_{2}$ & $-0,238^{*}$ \\
\hline$B_{22}$ & $0,263^{*}$ \\
\hline$B_{12}$ & 0,914 \\
\hline
\end{tabular}

*Os coeficientes não foram significativos a um nível de confiança de $95 \%$.

O dia diagrama de Pareto foi utilizado na análise e cálculo dos efeitos de cada parâmetro. Trata-se de uma representação gráfica onde podemos analisar os efeitos de um dado experimento [8]. A partir dele podemos obter as melhores interações entre os fatores e quais destes são mais significativos no experimento. $\mathrm{O}$ valor $\mathrm{p}=0,05$ indica o limite de significância, abaixo do qual a variável pode ser considerada insignificante ou sem influência no experimento a um nível de significância de 95\%

Conforme mostrado no gráfico de Pareto da Figura 1 e na tabela da ANOVA através do p-valor maior que 0,05 [8], nem todos os efeitos foram significativos a um nível de confiança de $95 \%$.

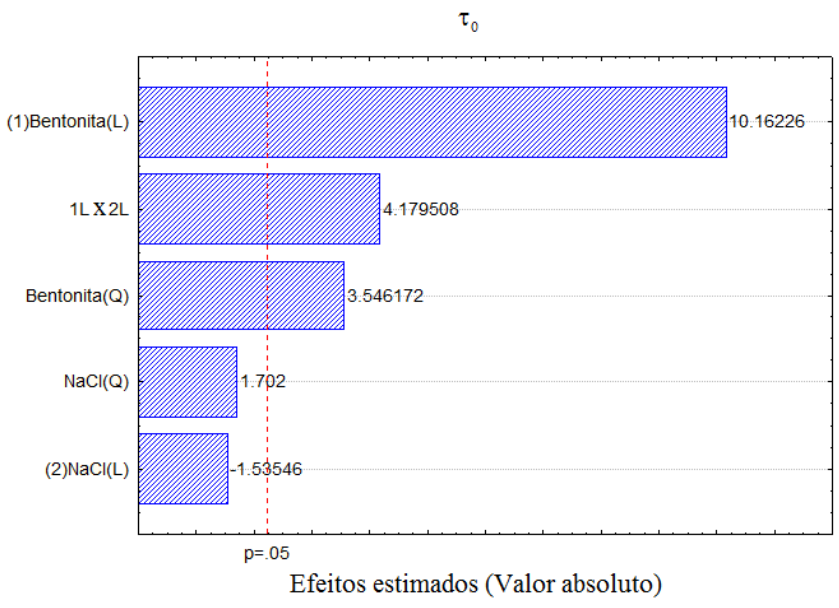

Figura 1: Diagrama de Pareto dos efeitos das variáveis independentes sobre o limite de escoamento.

O coeficiente de determinação $\left(\mathrm{R}^{2}=0,932\right)$ mostra que a Equação 7 está bem ajustada. Os efeitos da concentração do cloreto de sódio (linear e quadrático) não foram significativos, embora a sua interação com a concentração de bentonita tenha sido. A superfície de resposta dessa variável é mostrada na Figura 2. 


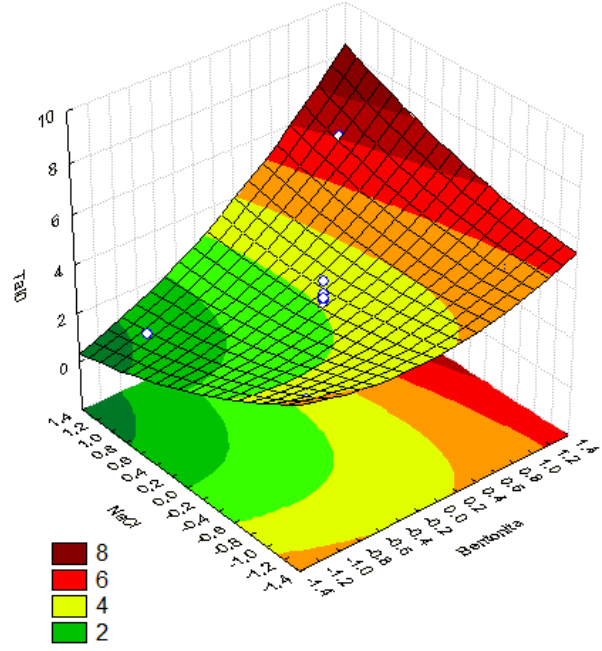

Figura 2: Superfície de resposta para o limite de escoamento em função da concentração de bentonita e da concentração de cloreto de sódio.

Ao analisarmos a superfície de resposta da Figura 2, percebemos que o limite de escoamento aumenta com a concentração de bentonita e com a concentração do cloreto de sódio e atinge um ponto de mínimo quando a concentração do cloreto de sódio é máxima e a concentração de bentonita é mínima. As faixas de concentração analisadas de bentonita sódica e de cloreto de sódio são de $1,34(-1,4142)$ a 12,66\% $(+1,4142)$ (em massa) e de 5,76 (-1,4142) a 14,24\% (+1,4142) (em massa) respectivamente.

Fluidos argilosos com altas concentrações de bentonita possuem valores de tensão limite de escoamento muito superiores do que aqueles exibidos por fluidos poliméricos com pouca ou nenhuma concentração de argila [2], o que justifica a análise desse parâmetro nesta etapa.

Um aumento da concentração de bentonita gera uma maior força de coesão entre as partículas. O aumento no valor do limite de escoamento por conta do aumento da concentração de bentonita é explicado devido ao fato de ser necessária uma maior tensão para iniciar o escoamento para quebrar a rede formada pelas partículas do argilomineral quanto maior for a concentração do mesmo, ou seja, maior deverá ser a tensão para reorientar as partículas de argilomineral para o escoamento [9].

Se fossemos analisar sob a perspectiva da dupla camada elétrica, o aumento da concentração do eletrólito na dispersão achata a dupla camada elétrica permitindo a aproximação de uma partícula de argilomineral com a outra a ponto de haver a floculação do sistema, logo, deveria aumentar as propriedades reológicas da suspensão, incluindo a tensão limite de escoamento. Os dados obtidos, no entanto, mostraram que isso ocorre apenas em altas concentrações de cloreto de sódio, pois sob condições de baixas concentrações de $\mathrm{NaCl}$, a presença do mesmo causa uma redução do $\tau_{0}$. Essa redução do limite de escoamento a baixas concentrações ocorre devido à redução das forças atrativas entre as partículas de argilominerais uma vez que o cloreto de sódio começa a ser adicionado ao sistema [10].

Ao que tudo indica, a partir de um determinado ponto, o aumento da concentração do eletrólito e contínuo achatamento da dupla camada elétrica permite a organização das lamelas de argila na forma de agregados, indisponibilizando área do argilomineral para interação e reduzindo as propriedades reológicas da dispersão [11]. Isso é condizente com o que LEWIS e THOMPSON [10] mostraram através de experimentos, nos quais ficou claro que o comportamento do eletrólito no meio dependerá da concentração do argilomineral disperso.

\subsection{Análise do índice de consistência $(k)$}

A Tabela 6 apresenta os valores da análise de variância (ANOVA) para a variável de resposta índice de consistência $(k)$, onde também é apresentado o valor do coeficiente de determinação $\left(\mathrm{R}^{2}\right)$. 
Tabela 6: Análise de variância (ANOVA) para a variável índice de consistência $(k)$ relativa ao PCC.

\begin{tabular}{l|l|l|l|l|l}
\hline Fator analisado & Soma dos Quadrados & Graus de Liberdade & Quadrado Médio & F & p \\
\hline Bentonita (Linear) & 3,603 & 1 & 3,603 & 164,587 & 0,000 \\
\hline Bentonita (Quad.) & 0,003 & 1 & 0,003 & 0,132 & 0,724 \\
\hline $\mathrm{NaCl}$ (Linear) & 0,420 & 1 & 0,420 & 19,186 & 0,001 \\
\hline $\mathrm{NaCl}$ (Quad.) & 0,106 & 1 & 0,106 & 4,848 & 0,049 \\
\hline Bentonita x NaCl & 0,069 & 1 & 0,069 & 3,185 & 0,105 \\
\hline Erro Puro & 0,161 & 7 & 0,023 & $\mathbf{R}^{\mathbf{2}}$ (máx.) & $\mathbf{R}^{\mathbf{2}}$ \\
\hline Falta de Ajuste & 0,058 & 3 & 0,019 & & \multirow{2}{*}{0,951} \\
\hline Total & 4,420 & 15 & - & 0,963 & \\
\hline
\end{tabular}

A partir de uma regressão multivariada, podemos chegar numa equação para calcular o valor de $k$ a partir das variáveis independentes codificadas na Equação 8.

$$
k=B_{0}+B_{1} B e n t+B_{11} \text { Bent }^{2}+B_{2} \mathrm{NaCl}+B_{22} \mathrm{NaCl}^{2}+B_{12} \text { Bent. NaCl }
$$

O valor de $k$ é dado em Pa.s ${ }^{\mathrm{n}}$. Os valores dos coeficientes são mostrados na Tabela 7.

Tabela 7: Coeficientes de regressão para a variável índice de consistência $(k)$.

\begin{tabular}{l|l}
\hline Coeficiente & Valor \\
\hline$B_{0}$ & 1,565 \\
\hline$B_{1}$ & 0,671 \\
\hline$B_{11}$ & $0,019^{*}$ \\
\hline$B_{2}$ & $-0,229$ \\
\hline$B_{22}$ & $-0,115$ \\
\hline$B_{12}$ & $-0,132 *$ \\
\hline
\end{tabular}

*Os coeficientes não foram significativos a um nível de confiança de $95 \%$.

Conforme mostrado no gráfico de Pareto da Figura 3 e na tabela da ANOVA através do p-valor maior que 0,05 [8] , nem todos os efeitos foram significativos a um nível de confiança de $95 \%$.

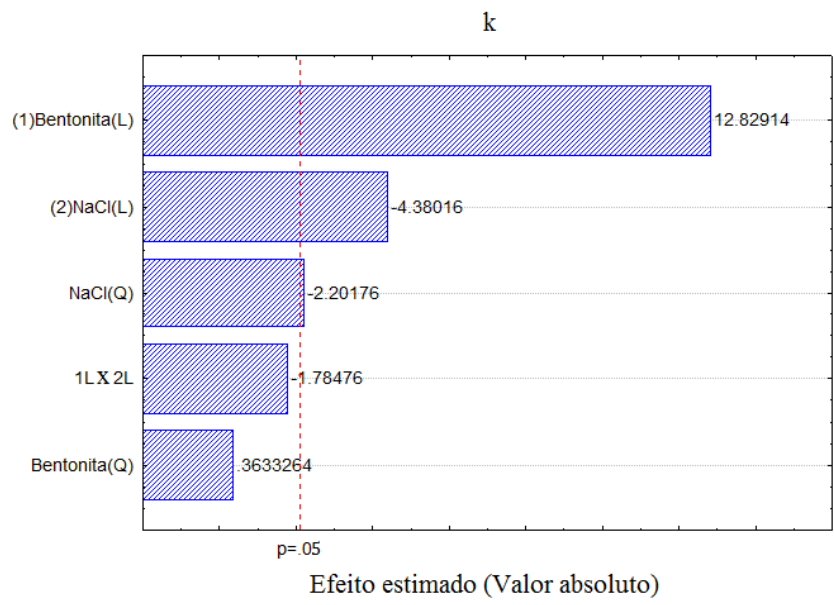

Figura 3: Diagrama de Pareto dos efeitos das variáveis independentes sobre o índice de consistência.

$\mathrm{O}$ coeficiente de determinação $\left(\mathrm{R}^{2}=0,951\right)$ mostra que a Equação 8 está bem ajustada. $\mathrm{O}$ efeito quadrático da concentração da bentonita e o efeito da interação da bentonita com o cloreto de sódio não foram 
significativos. A superfície de resposta dessa variável é mostrada na Figura 4.

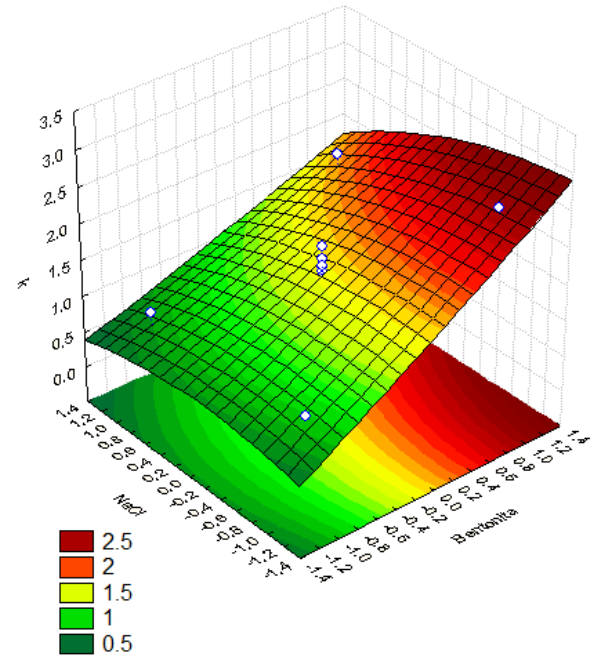

Figura 4: Superfície de resposta para o índice de consistência em função da concentração de bentonita e da concentração de cloreto de sódio.

A Figura 4 mostra que o índice de consistência aumenta com a concentração de bentonita de maneira linear e diminui com a concentração de cloreto de sódio. O valor de $\mathrm{k}$ atinge o seu valor máximo quando a concentração do cloreto de sódio é mínima e a concentração de bentonita é máxima. As faixas de concentração analisadas foram as mesmas do limite de escoamento: bentonita sódica de $1,34(-1,4142)$ a 12,66\% $(+1,4142)$ (em massa)e de cloreto de sódio de $5,76(-1,4142)$ a 14,24\% $(+1,4142)$ (em massa).

A explicação para o comportamento do índice de consistência deve ser explicada pelo comportamento dos dois viscosificantes utilizados nas amostras: a bentonita sódica e a goma xantana.

No que tange à bentonita, a interpretação desses resultados é similar a do limite de escoamento. Uma vez que o índice de consistência indica o grau de resistência ao escoamento do fluido, uma vez que a concentração de bentonita aumenta, a quantidade de partículas de argilominerais aumenta, assim como as suas interações.

No momento em que aumentamos a concentração do eletrólito, a organização dos argilominerais é alterada na medida em que a distribuição de cargas entre as partículas também é alterada. As forças repulsivas aumentam e a resistência ao fluxo das partículas diminuem.

Pela perspectiva do viscosificante polimérico, as propriedades reológicas de dispersões com goma xantana são altamente dependentes da concentração do polímero e de qualquer eletrólito adicionado. Segundo Borges e Vendruscolo [12], a adição de eletrólitos ao sistema reduz o valor dos parâmetros reológios da dispersão e isso pode ser atribuído ao resultado da mudança na conformação do polímero, causando um decréscimo no volume hidrodinâmico do mesmo.

\subsection{Análise do índice de comportamento (n)}

A Tabela 8 apresenta os valores da análise de variância (ANOVA) para a variável de resposta índice de comportamento $(n)$, onde também é apresentado o valor do coeficiente de determinação $\left(\mathrm{R}^{2}\right)$. 
Tabela 8: Análise de variância (ANOVA) para a variável índice de comportamento $(n)$ relativa ao PCC.

\begin{tabular}{l|l|l|l|l|l}
\hline Fator analisado & Soma dos Quadrados & Graus de Liberdade & Quadrado Médio & F & p \\
\hline Bentonita (Linear) & 0,005 & 1 & 0,005160 & 164,587 & 0,000 \\
\hline Bentonita (Quad.) & 0,014 & 1 & 0,014253 & 0,132 & 0,000 \\
\hline $\mathrm{NaCl}$ (Linear) & 0,003 & 1 & 0,003 & 19,186 & 0,004 \\
\hline $\mathrm{NaCl}$ (Quad.) & 0,012 & 1 & 0,012 & 4,848 & 0,000 \\
\hline Bentonita x NaCl & 0,000 & 1 & 0,000 & 3,185 & 0,224 \\
\hline Erro Puro & 0,001 & 7 & 0,000 & $\mathbf{R}^{\mathbf{2}}$ (máx.) & $\mathbf{R}^{\mathbf{2}}$ \\
\hline Falta de Ajuste & 0,001 & 3 & 0,000 & & \multirow{2}{*}{0,947} \\
\hline Total & 0,037 & 15 & - & 0,982 & \\
\hline
\end{tabular}

A partir de uma regressão multivariada, podemos chegar numa equação para calcular o valor de $n$ a partir das variáveis independentes codificadas na Equação 9.

$$
n=B_{0}+B_{1} B e n t+B_{11} B_{e n t}^{2}+B_{2} \mathrm{NaCl}+B_{22} N a C l^{2}+B_{12} \text { Bent. NaCl }
$$

O valor de $n$ é adimensional. Os valores dos coeficientes são mostrados na Tabela 9.

Tabela 9: Coeficientes de regressão para a variável índice de comportamento $(n)$.

\begin{tabular}{l|l}
\hline Coeficiente & Valor \\
\hline$B_{0}$ & 0,357 \\
\hline$B_{1}$ & $-0,025$ \\
\hline$B_{11}$ & 0,042 \\
\hline$B_{2}$ & 0,018 \\
\hline$B_{22}$ & 0,040 \\
\hline$B_{12}$ & $0,009^{*}$ \\
\hline
\end{tabular}

*Os coeficientes não foram significativos a um nível de confiança de $95 \%$.

Conforme mostrado no gráfico de Pareto da Figura 5 e na tabela da ANOVA através do p-valor maior que 0,05 [8], nem todos os efeitos foram significativos a um nível de confiança de $95 \%$.

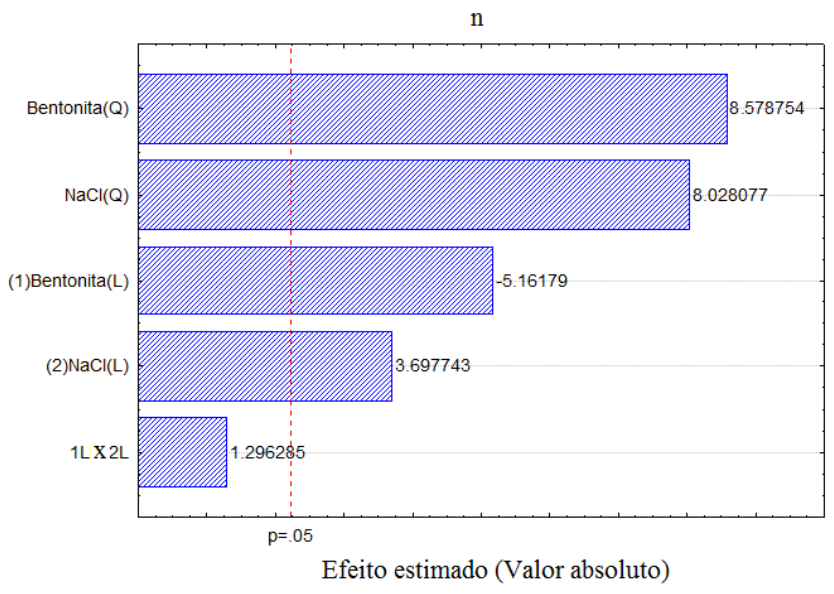

Figura 5: Diagrama de Pareto dos efeitos das variáveis independentes sobre o índice de comportamento.

O coeficiente de determinação $\left(\mathrm{R}^{2}=0,947\right)$ mostra que a Equação 9 está bem ajustada. $\mathrm{O}$ único efeito que não foi significativo para esse parâmetro foi o efeito da interação da concentração da bentonita com o 
cloreto de sódio. A superfície de resposta dessa variável é mostrada na Figura 6.

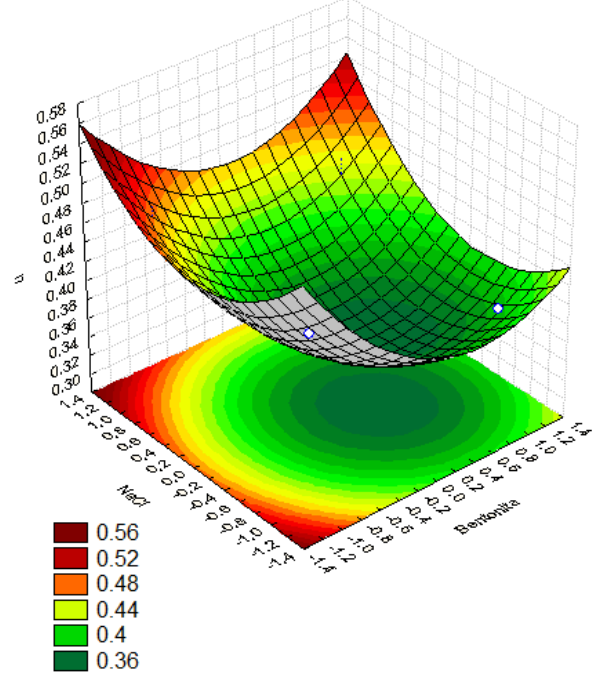

Figura 6: Superfície de resposta para o índice de comportamento em função da concentração de bentonita e da concentração de cloreto de sódio.

Ao analisarmos a Figura 6, percebemos que há um ponto de mínimo no valor de $n$ a depender da concentração de bentonita e da concentração de cloreto de sódio. Mais uma vez, as faixas de concentração analisadas foram as mesmas dos dois parâmetros anteriores: bentonita sódica de $1,34(-1,4142)$ a $12,66 \%$ $(+1,4142)$ (em massa) e de cloreto de sódio de $5,76(-1,4142)$ a $14,24 \%(+1,4142)$ (em massa).

Para determinarmos esse ponto de mínimo [8], derivamos a Equação 9 em relação à concentração de bentonita e à concentração de cloreto de sódio e igualamos ambas as equações a zero, conforme mostrado nas Equações 10 e 11.

$$
\begin{aligned}
& \frac{\partial \mathrm{n}}{\partial \mathrm{NaCl}}=B_{2}+2 B_{22} \mathrm{NaCl}+B_{12} \mathrm{Bent}=0 \\
& \frac{\partial \mathrm{n}}{\partial \mathrm{Bent}}=B_{1}+2 B_{11} \mathrm{Bent}+B_{12} \mathrm{NaCl}=0
\end{aligned}
$$

No entanto, como mostrado na Figura 5, o coeficiente $B_{12}$ não foi significativo em um nível de confiança de $95 \%$, ou seja, a interação entre as variáveis concentração de cloreto de sódio e concentração de bentonita não é significativa no modelo, e, portanto, iremos desprezá-la. Desta forma, as Equações 10 e 11 se resumem ao seguinte sistema de Equações:

$$
\begin{aligned}
& B_{2}+2 B_{22} \mathrm{NaCl}=0 \\
& B_{1}+2 B_{11} \text { Bent }=0
\end{aligned}
$$

Substituindo os valores dos coeficientes, chegamos nos valores: $\mathrm{NaCl}=-0,23(9,4 \%$ em massa) e Bent $=0,30(7,9 \%$ em massa).

Como dito anteriormente, o valor do índice de fluxo indica o grau de pseudoplasticidade do fluido, ou seja, o quanto o fluido "afina" com o aumento da taxa de cisalhamento. Quanto menor o valor de $n$, menor é o valor da viscosidade aparente com o aumento da taxa de cisalhamento, ou seja, menor resistência o fluido apresenta para escoar [3,5].

Se analisarmos a superfície de resposta da Figura 6, existe uma composição de bentonita e cloreto de sódio que faz com que o valor de $n$ chegue a um mínimo. A pseudoplasticidade para dispersões argilosas pode ser explicada pela mudança de orientação dos argilominerais com o aumento da taxa de cisalhamento. $\mathrm{Na}$ ausência de cisalhamento ou sob baixas taxas, as partículas de argilominerais estarão preferencialmente orientadas em formato de rede, nas quais as interações entre as folhas serão do tipo face-face (agregadas) ou face-aresta (floculadas). A partir do momento em que a taxa de cisalhamento aumenta, as folhas dos argilo- 
minerais tenderão a se orientar de maneira que facilite o fluxo, diminuindo a resistência ao movimento [13].

No momento em que eletrólitos são adicionados ao sistema, a orientação dos argilominerais muda, de modo que a sua pseudoplasticidade também acompanha essa mudança. Para a bentonita sódica e o cloreto de sódio analisados dentro da faixa de estudo, as concentrações nas quais o índice de fluxo apresenta o menor valor (em torno de 0,35$)$ é de aproximadamente $-0,23(9,4 \%$ em massa) para o cloreto de sódio e $0,3(7,9 \%$ em massa) para a bentonita.

Uma explicação razoável para esse comportamento do $n$ é que, a partir de determinadas concentrações de bentonita e $\mathrm{NaCl}$, a mudança de orientação das partículas ocorre de maneira mais fácil com o aumento da taxa de cisalhamento. Alterações nas concentrações de bentonita ou cloreto de sódio a partir desse ponto de mínimo causariam um aumento no índice de comportamento [7].

\subsection{Validação dos modelos}

Com o objetivo de validar os modelos gerados para a tensão limite de escoamento e os índices de consistência e de fluxo, escolheu-se ao acaso um ponto dentro da superfície de resposta gerada e preparou-se o fluido com as variáveis escolhidas. As variáveis independentes escolhidas ao acaso para a preparação do fluido bem como os seus valores reais e codificados encontram-se na Tabela 10.

Tabela 10: Valores das variáveis independentes escolhidas ao acaso para a validação dos modelos

\begin{tabular}{l|l|l}
\hline Variável & Valor da variável & Variável codificada \\
\hline Bentonita sódica & $7,69 \%$ & $+0,23$ \\
\hline Cloreto de sódio & $9,64 \%$ & $-0,12$ \\
\hline
\end{tabular}

Os valores medidos e calculados, bem como o erro relativo em relação ao valor medido é apresentado na Tabela 11. Os valores calculados foram obtidos a partir das Equações 7, 8 e 9 enquanto que os valores medidos foram obtidos experimentalmente seguindo a mesma metodologia apresentada no item 2.2.

Tabela 11: Valores dos parâmetros reológicos obtidos e o erro relativo entre os valores medidos e calculados.

\begin{tabular}{l|l|l|l}
\hline Variável dependente & Medido & Calculado & Erro relativo (\%) \\
\hline$\tau_{0}$ & 3,096 & 3,599 & 16,25 \\
\hline$k$ & 1,5147 & 1,7583 & 16,08 \\
\hline$n$ & 0,3504 & 0,3514 & 0,30 \\
\hline
\end{tabular}

Os erros de $16,25 \%, 16,08 \%$ e $0,3 \%$ para os valores de $\tau_{0}, k$ e $n$ respectivamente, mostram que os modelos obtidos representam bem os valores dos parâmetros para a região estudada.

\section{CONCLUSÕES}

Os resultados apresentados nesse estudo permitem concluir que as concentrações de bentonita sódica e de cloreto de sódio são variáveis que influenciam de modo significativo os parâmetros reológicos de dispersões argilosas. Uma vez que o planejamento experimental utilizado trabalha com altas concentrações de bentonita, o modelo reológico utilizado foi o de Herschel-Bulkley cujos parâmetros são a tensão limite de escoamento $\left(\tau_{0}\right)$, o índice de consistência $(k)$ e o índice de comportamento $(n)$.

O aumento na concentração de bentonita para quase toda a faixa estudada mostrou um aumento no limite de escoamento. Já o aumento da concentração de cloreto de sódio mostrou uma diminuição do valor de $\tau_{0}$ para pequenas concentrações de bentonita e um aumento no valor dessa variável para altas concentrações de bentonita.

$\mathrm{O}$ valor de $k$ aumenta com a concentração de bentonita e, de um modo geral, diminui com o valor da concentração de cloreto de sódio. Já em relação ao valor de $n$, observou-se um ponto de mínimo na superfície de resposta analisada. A depender das concentrações de cloreto de sódio e de bentonita, haverá um arranjo da dispersão que provocará uma maior ou menor pseudoplasticidade. Para a região analisada, o valor de mínimo de $n(0,35)$ ocorre para as concentrações de $9,4 \%$ para o cloreto de sódio e de $7,9 \%$ para a bentonita (ambas as porcentagens em massa). 
O estudo deixou claro, portanto, que é possível a manipulação das concentrações da argila e dos eletrólitos para se obter parâmetros reológicos que melhorem a performance do fluido de perfuração, evitando a decantação de cascalhos no fundo do poço durante a estática, melhorando o transporte dos cascalhos do fundo do poço até a superfície e minimizando as perdas de carga durante a circulação do fluido no poço.

\section{BIBLIOGRAFIA}

[1] THOMAS, J. E., Fundamentos de engenharia de petróleo, 2 ed., Rio de Janeiro, Interciência, 2004.

[2] GRAY, G. R., DARLEY, H.C.H., CAENN, R., Fluidos de perfuração e completação, 6 ed., Rio de Janeiro, Elsevier, 2014.

[3] PETROBRAS, Manual de Fluidos, 1 ed., Rio de Janeiro, 2011.

[4] MI SWACO, Engineering Drilling Fluids Manual, 1 ed., Texas - US, 2001.

[5] MACHADO, J.C.V., Reologia e escoamento de fluidos: Ênfase na indústria do petróleo, 1 ed., Rio de Janeiro, Interciência, 2002.

[6] SHIROMA, P. H., Estudo do comportamento reológico de suspensões aquosas de bentonita e CMC: influência da concentração do NaCl, Dissertação de M.Sc., USP, São Paulo, SP, Brasil, 2012.

[7] SOUZA, G. S., Caracterização reológica de dispersões argilosas com goma xantana para fluidos de perfuração de poços de petróleo: Influência de sais monovalentes, bivalentes e da temperatura, Dissertação de M.Sc., PPEQ/UFBA, Salvador, BA, Brasil, 2016.

[8] BARROS NETO, B., SCARMINIO I. S., BRUNS, R. E., Como fazer experimentos: Pesquisa e desenvolvimento na ciência e na indústria, 2 ed., Campinas - SP, Unicamp, 2003.

[9] CHOO, K. Y.; BAI, K. "Effects of bentonite concentration and solution ph on therheological properties and long-term stabilities of bentonite suspensions”, Applied Clay, v. 108,, pp. 182-190, 2015.

[10] LEWIS, W.; THOMPSON, W. "Colloidal Properties of Clay Suspensions." 1934. Tulsa Meeting.

[11] NASH, V. "Role of Exchangeable cations in viscosity of clay suspensions", Applied Clay, Tulsa, Oklahoma 1990.

[12] BORGES, C. D., VENDRUSCOLO, C. T. "Comportamento reológico de xantana produzida por xanthomonas arborícola pv pruni para aplicação em fluido de perfuração de poços de petróleo", Polímeros, v. 19,, pp. 160-165, 2009.

[13] NASCIMENTO, R., AMORIM, L. "Desenvolvimento de fluidos aquosos com bentonita para perfuração de poços de petróleo onshore”, Cerâmica, v. 56, pp. 179-187, 2010. 